\title{
Sentence length and sentence retention in the free-learning situation'
}

\author{
EDWIN MARTIY AND KELYN H. ROBERTS \\ UNIVERSITY OF MICHIGAN
}

The hypothesis that sentence length is not a significant factor in sentence recall was tested and rejected. The lengths studied were 5,7 , and 9 words. All sentences were of the active-affirmative type. A structural-complexity factor was found to play no role in recall.

In previously reported research by the present authors on memory for English sentences, sentence length was fixed over all conditions at seven words. In the free-learning situation (Martin \& Roberts, 1966), it was. found that "kernels," that is, simple activeaffirmative sentences, were retained signiflcantly more poorly than other syntactic types, for example, passives. Contrary to this result, Mehler (1963), also using the free-learning technique, found kernels to be retained better than the other types. A possible explanation of Mehler's result is the fact that his kernels were shorter sentences than were the corresponding transformations.

The present experiment is designed to test the hypothesis that sentence length is not a variable of interest in sentence retention. The sentences used were all active-affirmative sentences of length 5,7 , or 9 words. The sentences also varied in structural complexity, or mean depth (Yngve, 1960; Martin \& Roberts, 1966; Martin, Roberts, \& Collins, in press). Sentence length was a between-S variable, mean depth a withinS variable.

\section{Method}

Elghteen active-affirmative sentences were constructed that ranged in mean depth value from 1.00 to 1.66. There were six each of word-lengths 5,7 , and 9. Thirty University of Michigan undergraduates volunteered for participation. Ten of these, five males and five females, were assigned to each of the three sentence-length conditions.

Each $S$ was instructed that he would hear six unrelated sentences read to him and that when he was told "go" he was to write out as many of them as he could remember. He was allowed to recall the sentences in any order he liked. Each recall was written on a separate $3 \times 5$ in. card, which was then turned face down. After the completion of recall, the same six sentences were read again, in a different order, and again recall was attempted. There were six such free-learning trials.

For each sentence-length condition, the six sentences were ordered over the six presentation trials such that each sentence occurred once in each position. The $E$ sat vis-a-vis the $S$ and read the sentences in immediate succession in an ordinary tone of voice.

\section{Results}

The trial by trial proportion of completely correct recalls are posted in Fig. 1, where each point summarizes (10 Ss) $\times(6$ sentences $)=60$ recall events. Analysis of variance indicates: (1) that sentence length was a highly significant variable $(F=10.17, \mathrm{df}=2 / 27$, $\mathrm{p}<.001$ ); (2) that trials was also highly significant $(F=51.33, d f=5 / 135, p<.001)$; and (3) that lengths and trials falled to interact significantly $(F=1.90$, df $=10 / 135, p>.05$ ). Thus the hypothesis that "sentence length is not a variable of interest in sentence retention" is rejected.

Of the (10 Ss) $\times(6$ sentences $) \times(6$ trials $)=360$ recall events per sentence-length condition, there were 114, 155, and 230 errors of one type or other for lengths 5 , 7 , and 9 , respectively. The proportion of these that were simple synonym substitutions are .26, .20, and .34 for the three lengths. This type error tended to increase with trials; collapsing over the three lengths, the proportion of all errors that were synonym substitutions, by trials, are $.09, .14, .18, .20, .19$, and .20 . All other error types decreased with trials. The most striking error type to decrease over trials was that of omis-

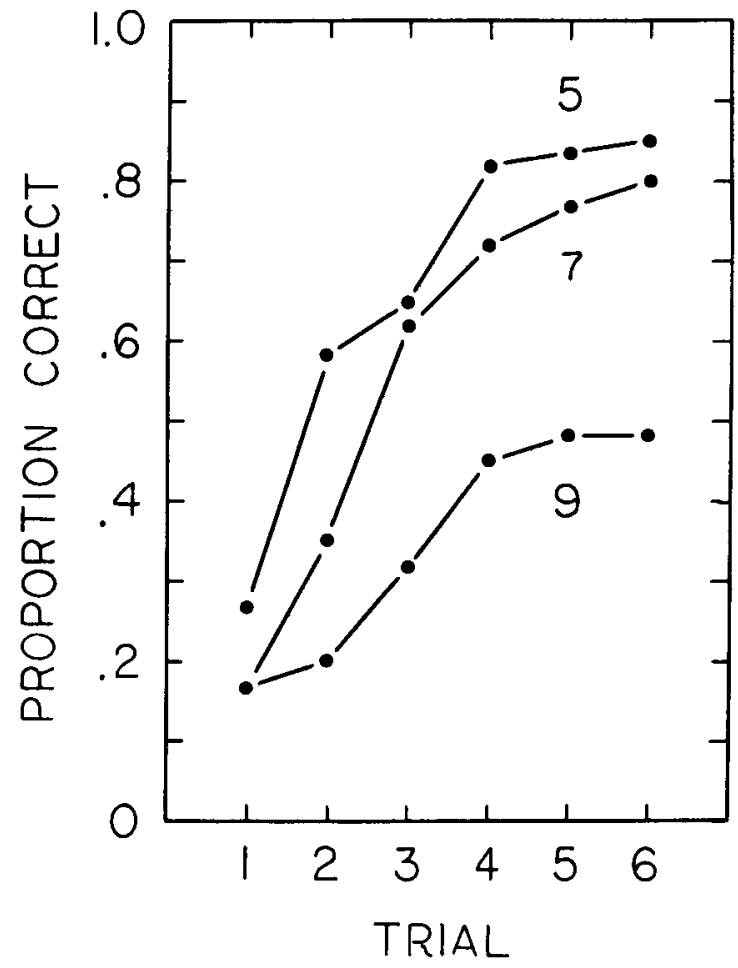

Fig. 1. Proportion completely correct recalls as a function of trial number. Parameter is length of sentence. 
sions: The trial by trial proportions, collapsing oversentence lengths, are .45, .26, .14, .07, .04, and .03. Of all the errors made for a given sentence length, the proportion that were omissions are .36, .25, and .30 for lengths 5,7 , and 9 , respectively.

Structural complexity failed to exert an effect. If the proportion correct recalls are plotted against mean depth, in a sentence by sentence fashion, it turns out that mean depth did not play a discernable role in recall and did not interact with sentence length. Discussion

The principal result is that sentence length is indeed a highly significant factor in sentence recall. This means that previous research on sentence retention wherein sentence length was confounded with such experimental variables as syntactic type may well require replication under conditions where length is controlled.

A systematic scrutiny of sentences used reveals that nouns, articles, and prepositions are the word classes that most increase in frequency with additional length. Adverbs and adjectives account for little, if any, of the effect. Earlier work by Martin, Roberts, \& Collins (in press) suggests that word class analyses may be a productive approach to the problems of behavioral linguistics. They demonstrated variability in processing different word classes, where nouns were a class of key importance. Thus the increase in noun frequency with longer sentences may imply additional, significant entries into memory, and hence underlie the poorer retention of longer sentences.
Among the six sentences of each of the lengths 5, 7, and 9 , there were, on the average, 1.5, 2.2, and 3.0 nouns per sentence.

The role of presented sentence mean depth in recall requires comment. Martin \& Roberts (1966) demonstrated that sentence complexity as indexed by mean depth significantly affects retention in the free-learning situation. In the present experiment, there is no detectable effect of complexity. Why this is the case is not clear to us, especially in view of the fact that significant mean depth effects have been observed also in the short-term memory situation (Martin, Roberts, \& Collins, in press; Roberts, 1966).

\section{References}

Martin, E., \& Roberts, K. H. Grammatical factors in sentence retention. J. verbal Learn. verbal Behav., 1966, 5, 211-218.

Martin, E., Roberts, K. H., \& Collins, A. M. Short-term memory for sentences. J. verbal Learn. verbal Behav., in press.

Mehler, J. Some effects of grammatical transformations on the recall of English sentences. J. verbal Learn. verbal Behav., 1963, 2, 346-351.

Roberts, $\mathbf{K} . \mathbf{H}$. The interaction of normative associations and grammatical factors in sentence retention. Paper read at Midwest. Psychol. Assoc., May, 1966.

Yngve, V. H. A model and an hypothesis for language structure. Proc. Amer. Phil. Soc., 1960, 104, 444-466.

\section{Note}

1. This research was supported by the Advanced Research Projects Agency, Department of Defense, monitored by the Air Force Office of Scientific Research, under Contract No. AF (638)-1235 with the Human Performance Center, Department of Psychology. The data were collected by Käthe E. Schultz. 